Portland State University

PDXScholar

\title{
Aryl Transfer Selectivity in Metal-free Reactions of Unsymmetrical Diaryliodonium Salts
}

David Stuart

Portland State University, dstuart@pdx.edu

Follow this and additional works at: https://pdxscholar.library.pdx.edu/chem_fac

Part of the Chemistry Commons

Let us know how access to this document benefits you.

\section{Citation Details}

Stuart, D. R. (2017). Aryl Transfer Selectivity in Metal-Free Reactions of Unsymmetrical Diaryliodonium Salts. Chemistry-A European Journal.

This Post-Print is brought to you for free and open access. It has been accepted for inclusion in Chemistry Faculty Publications and Presentations by an authorized administrator of PDXScholar. Please contact us if we can make this document more accessible: pdxscholar@pdx.edu. 


\section{CHEMISTRY A European Journal}

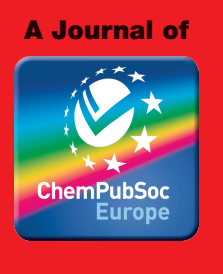

\section{Accepted Article}

Title: Aryl Transfer Selectivity in Metal-Free Reactions of Unsymmetrical Diaryliodonium Salts

Authors: David Ross Stuart

This manuscript has been accepted after peer review and appears as an Accepted Article online prior to editing, proofing, and formal publication of the final Version of Record (VoR). This work is currently citable by using the Digital Object Identifier (DOI) given below. The VoR will be published online in Early View as soon as possible and may be different to this Accepted Article as a result of editing. Readers should obtain the VoR from the journal website shown below when it is published to ensure accuracy of information. The authors are responsible for the content of this Accepted Article.

To be cited as: Chem. Eur. J. 10.1002/chem.201702732

Link to VoR: http://dx.doi.org/10.1002/chem.201702732 


\section{Aryl Transfer Selectivity in Metal-free Reactions of Unsymmetrical Diaryliodonium Salts}

David R. Stuart ${ }^{*[a]}$

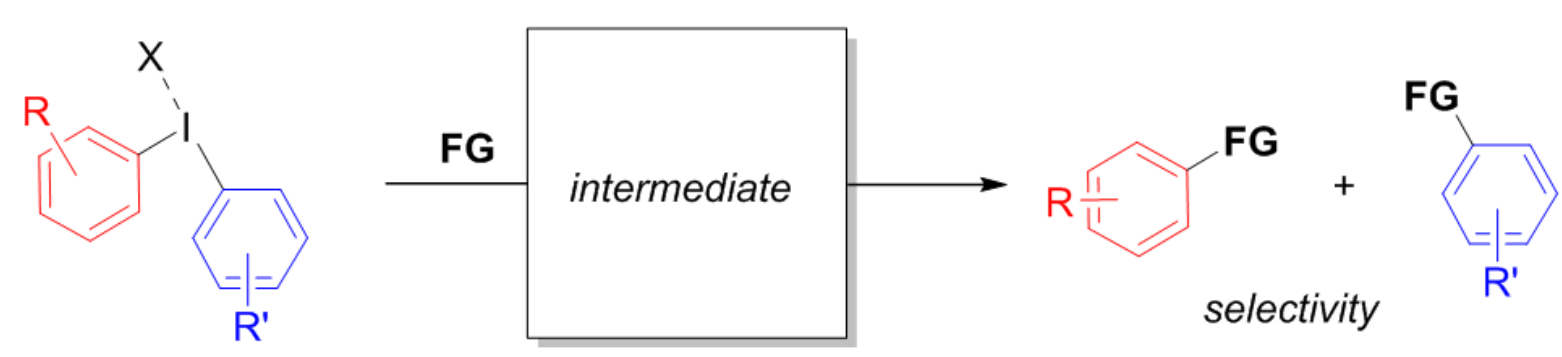

Intermediates

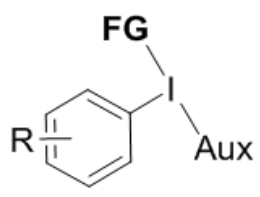

Electronic effects
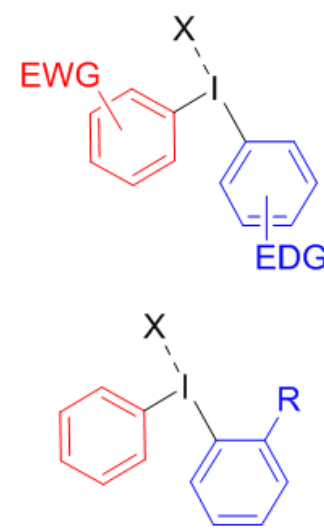

Steric effects

Intermediates

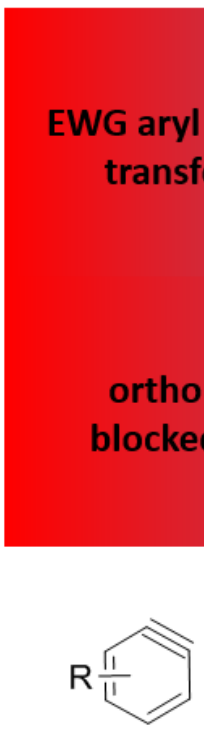

$\lambda^{3}$-iodane

aryne

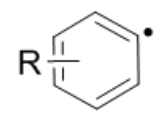

aryl radical

modest

electronic effect

anti-ortho and ortho effect

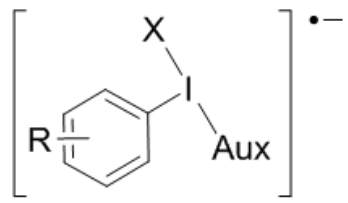

$\lambda^{3}$-iodane radical anion

EDG aryl group transfers

Modest anti-ortho and ortho effect 
Abstract: Aromatic rings are found in a wide variety of products, including pharmaceuticals, agrochemicals, and functional materials. Diaryliodonium salts are novel reagents used to transfer aryl groups under both metal-free and metal-catalyzed reactions and thereby synthesize arene-containing compounds. This review focuses on recent studies in selective aryl transfer reactions from unsymmetrical diaryliodonium salts under metalfree conditions. Reactions reported from 2008 to 2017, which represents a period of significant growth in diaryliodonium salt chemistry, are presented and organized by the type of reactive intermediate formed in the reaction. Specifically, reactions involving $\lambda^{3}$-iodane, $\lambda^{3}$-iodane radical anions, aryl radicals, and arynes are discussed. Chemoselectivity trends in aryl transfer are compared and contrasted across reaction intermediates and translation to potential auxiliaries are posited.

\section{Introduction}

Diaryliodonium salts ${ }^{[1]}$ are established aryl-transfer reagents with potential impact across diverse applications that include medicine, agriculture, technology, and energy. The use of these reagents to couple an aryl moiety to an incoming functional group (eq. 1) under metal-free or metal-catalyzed conditions has risen sharply in the past decade (Figure 1). ${ }^{[2]}$ This is arguably due to an increase in commercial and synthetic access to symmetrical and unsymmetical diaryliodonium salts that occurred prior to and during this period. ${ }^{[3]}$ Unsymmetrical aryl(auxiliary)iodonium salts (eq. 1) offer potential advantages over symmetrical ones in both their synthesis and applications. However, the primary challenge to overcome with unsymmetrical diaryliodonium reagents is chemoselective transfer of one aryl group over the other in a given functionalization reaction. We have been captivated by the potential of metal-free reactions with these reagents, and in particular the development of aryl(auxiliary)iodonium salts that address issues of selectivity (eq. 1). Several excellent books and review articles are available on the fundamental chemistry and applications of diaryliodonium salts, ${ }^{[1]}$ including a general discussion of chemoselective aryl transfer before $2008,{ }^{[1 \mathrm{~b}]}$ and the broader field of hypervalent iodine chemistry. ${ }^{[4]}$ This mini-review will specifically detail observations of selective aryl transfer from unsymmetrical salts in metal-free reactions between 2007-2017. The aim of this mini-review is three-fold: 1) collate the recent literature on metal-free aryl transfer from unsymmetrical diaryliodonium salts; 2) draw general conclusions regarding aryl transfer selectivity based on the nature of reactive intermediates formed; and 3) stimulate the discovery and development of general aryl(auxiliary)iodonium salt reagents for application in diverse synthetic settings.

[a] Prof. Dr. David R. Stuart

Department of Chemistry

Portland State University

1719 SW $10^{\text {th }}$ Ave, Portland, OR 97201, USA

E-mail: dstuart@pdx.edu

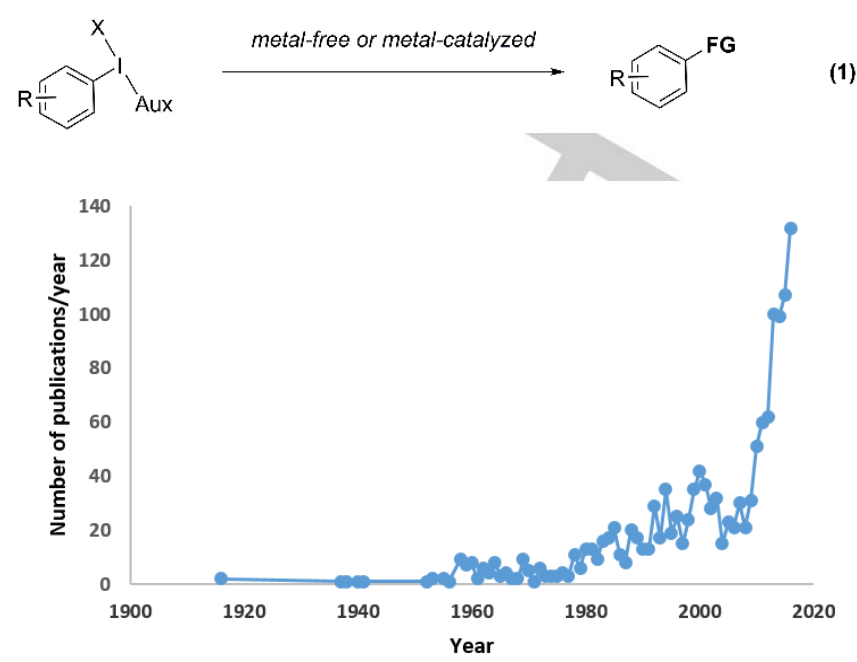

Figure 1. "Diaryliodonium salts" in the chemical literature. ${ }^{[1]}$

The breadth of retrosynthetic disconnections that are emerging with diaryliodonium salts is paramount to their popularity in metal-free reactions. Aryl cation equivalents, radical anions, aryl radicals, and arynes are accessible synthetic intermediates from diaryliodonium salts and participate in a broad range of $\mathrm{C}-\mathrm{C}$ and $\mathrm{C}$-heteroatom bond forming events, each with unique considerations for aryl transfer selectivity (Scheme 1). As such, the design and implementation of general auxiliaries on unsymmetrical diaryliodonium salts is only possible with a better understanding of inherent selectivity of aryl group transfer. A greater number of literature reports have appeared than could possibly be included in this mini-review and therefore only selected examples that report quantitative data on aryl transfer selectivity are explicitly discussed. Additionally, attempts have been made to provide a balanced overview of selectivity for each transformation, and the most selective examples are not always included. Readers are encouraged to consult the primary literature for additional examples and the breadth of selectivity possible. Given the diversity of intermediate and functional group combinations, this review is organized primarily by the types of reactive intermediates presented in Scheme 1. As a secondary consideration, and where possible (e.g., in $\lambda^{3}$-iodanes), the type of functional group/nucleophile that is arylated is included. There are two caveats to this organizational scheme. First, the literature discussed in each section is based on the intermediate proposed by the original authors. Second, many of the reactions discussed are new and have not been rigorously characterized mechanistically; therefore, it is possible in some cases that product ratios representing aryl transfer selectivity are the result of competing reaction pathways and more than one intermediate. 
Suggestions regarding the potential properties of aryl auxiliaries that could function as broadly applicable dummy ligands on aryl(auxiliary)iodonium salts are put forth for each of the intermediates discussed. Finally, as an explanation of the convention taken here, the diaryliodonium salt structures are drawn to emphasize their dynamic nature. Specifically, the bond between iodine and the X-group (or FG/Nu) is drawn as a long dashed line to indicate the participation of this group in the hypervalent bond in two different T-shaped conformations (vide infra).

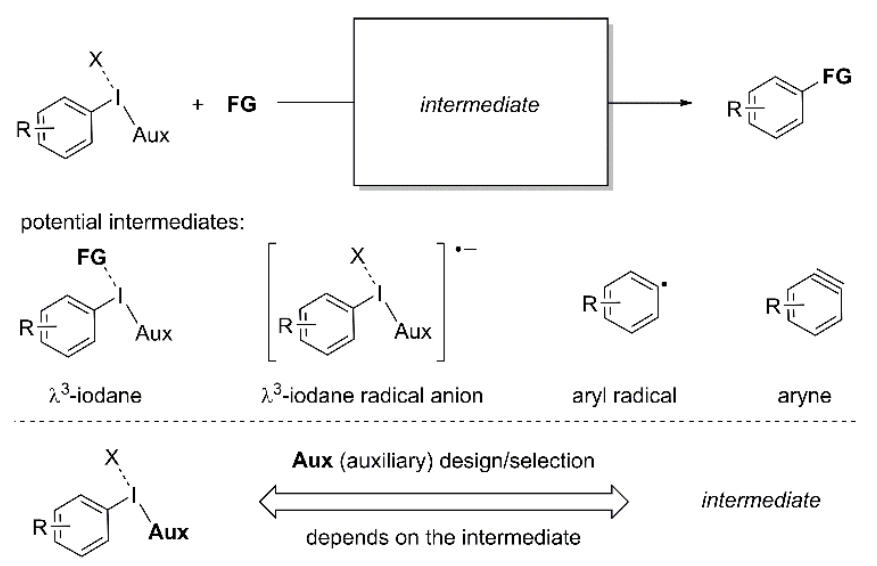

Scheme 1. Mechanistic pathways for aryl functionalization with diaryliodonium salts.

David Stuart was born and raised in Victoria, British Columbia, Canada. He obtained a B.Sc. (Honors) degree from the University of Victoria in 2005 and a Ph.D. from the University of Ottawa in 2010 under the supervision of Prof. Keith Fagnou. Dave was an NSERC Postdoctoral Fellow with Prof. Eric Jacobsen at Harvard from 2010-2012. He began his independent career in the Department of Chemistry at Portland State University in the fall of 2012. His research group is interested in the discovery and development of novel arylation reactions with unsymmetrical diaryliodonium salts.

\section{Arylation involving $\lambda^{3}$-iodane intermediates}

Nucleophile arylation that proceeds through a $\lambda^{3}$-iodane intermediate is the most common application of diaryliodonium salts. In this case, two rapidly equilibrating $\lambda^{3}$-iodanes lead to two different products via reductive coupling of the nucleophile with the proximal aryl group (Scheme 2). The energy barrier between the two $\lambda^{3}$-iodanes is low and the product distribution is under Curtain-Hammett control. ${ }^{[2],[5]}$ As in many organic reactions, electronic and steric effects are the predominant controlling factors for chemoselective aryl transfer from $\lambda^{3}$-iodanes to nucleophiles. This section of the review outlines how each of these effects influences aryl transfer selectivity and their confluence with nucleophile identity.

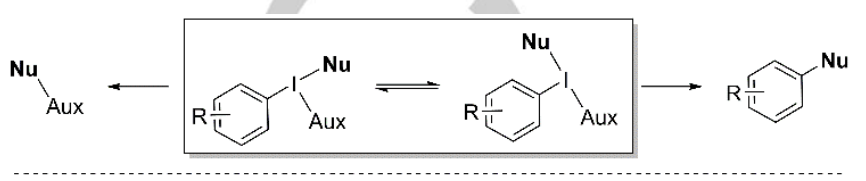
Electronic effects

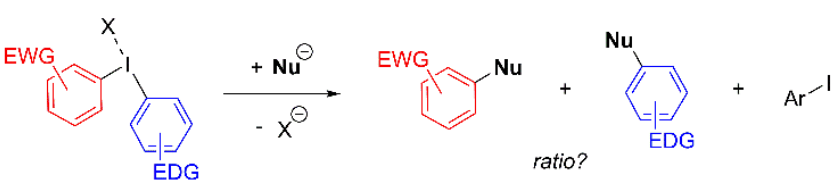

Steric effects<smiles>[R]c1ccccc1I([X])c1ccccc1</smiles><smiles>[X][N+](=O)[O-]</smiles><smiles>[R]c1ccccc1N</smiles>
$+\stackrel{\mathrm{Nu}}{+} \mathrm{Ar}^{-1}$

Scheme 2. Potential product distribution from $\lambda^{3}$-iodane intermediates and general effects of the aryl groups.

\subsection{Historical perspective on selective aryl transfer}

Early examples of aryl transfer selectivity based on electronic ${ }^{[6]}$ and steric ${ }^{[7]}$ effects were provided by Beringer and independently by Okawara and Wiegand, respectively (Scheme 3). Beringer noted that when unsymmetrical diaryliodonium salt 1 was refluxed in water with sodium hydroxide, 2-nitrophenol 2 (76\%) was obtained; the corresponding yield of phenyl iodide was not reported. Though the yield of both aryl fragments was not reported the high yield of 2-nitrophenol suggests electronic preference for ligand coupling of the hydroxide nucleophile with the more electron-deficient nitro-substituted aryl group. Wiegand provided further support for electronically controlled ligand coupling. ${ }^{[7 b]}$ The pyrolysis of $\mathbf{3}$ yielded a mixture of phenyl bromide $\mathbf{4}$ and 4-tolyl bromide 5 (Scheme 3). Despite the modest ratio (1.7: 1), preference for ligand coupling of bromide with the more electron-deficient aryl group (phenyl) was observed. In their work, Lancer and Wiegand also provided an example of the "ortho effect in which steric effects promote ligand coupling. ${ }^{[7 b]}$ In this particular case, pyrolysis of 6 a produced a mixture of phenyl bromide 7 and 2-tolyl bromide 8 (Scheme 3); reductive ligand coupling with the nucleophile was favored for the more sterically encumbered aryl group. The "ortho" effect may also have contributed to preferential coupling of the 2-nitrophenyl moiety discussed above. These observations provide the basis for the following discussion of electronic and steric control over ligand coupling across a diverse set of nucleophiles. 


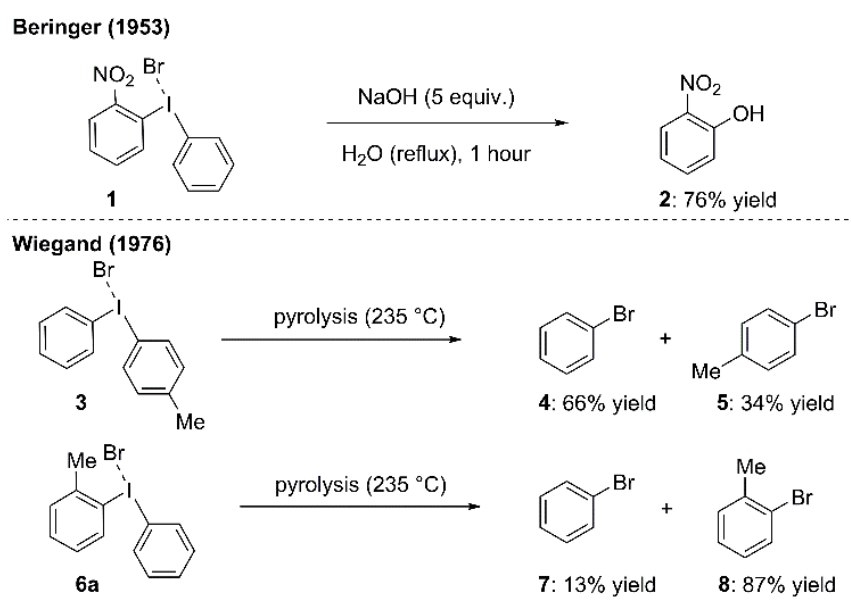

Scheme 3. Early observations of electronic and steric effects in aryl transfer.

\subsection{Boron nucleophiles}

Fernández and Muñiz demonstrated the sole example of metalfree boron arylation with diaryliodonium salts. ${ }^{[8]}$ In their pioneering work, the use of an acetate counter anion is critical for reactivity and the authors propose that it activates the diboron reagent and creates umpolung reactivity in the form of a boron nucleophile. The aryl transfer selectivity is modest in this transformation and is, in part, dependent on the diboron reagent (Scheme 4). When $\mathrm{B}_{2}$ (pin) $)_{2}$ and 9 are employed as the pronucleophile and electrophile, respectively, no aryl transfer selectivity is observed (i.e., 10 and $\mathbf{1 1}$ are formed in equal quantity; Scheme 4). However, when the mixed diboron reagent $B($ pin) $B($ dan) is used in a reaction with 9 , a slight preference $(1.7: 1)$ for transfer of the electron-deficient aryl group is observed (12:13, 1.7:1; Scheme 4). The steric effects on aryl transfer selectivity are more pronounced than electronic effects in this case. When (phenyl)(mesityl)iodonium acetate $\mathbf{1 4 a}$ is used, a small bias for transfer of the phenyl moiety is observed (i.e., 16: 17 1.6: 1). Additionally, when the more sterically hindered tri-isopropylphenyl group is incorporated into 15 the preference for phenyl transfer increases to 5.7: 1 (16:18; Scheme 4). Selectivity, for transfer of the less sterically hindered aryl group is opposite to Wiegand's work. ${ }^{[\mathrm{bb}]}$ This is not the first and only example where selectivity opposite to the "ortho" effect is observed. Accordingly, this is termed the "anti-ortho" effect. [9a]

\subsection{Carbon nucleophiles}

The arylation of carbon nucleophiles with diaryliodonium salts has historically involved soft nucleophiles, such as malonates. This section of the review, however, includes other enolate derivatives, acyl anion equivalents, and heteroarenes, as compatible carbon nucleophiles. The aryl transfer selectivity is presented in the context of electronic and steric effects of the aryl groups on the unsymmetrical diaryliodonium salts.

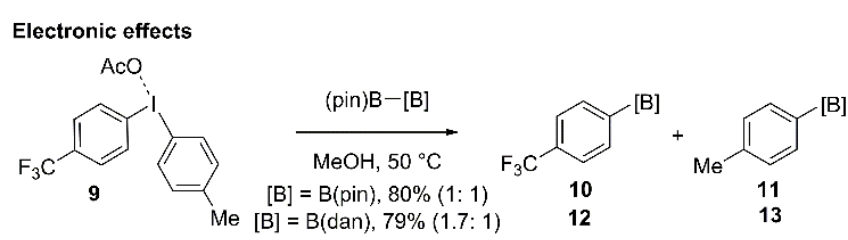

Steric effects

$\mathrm{AcO}$

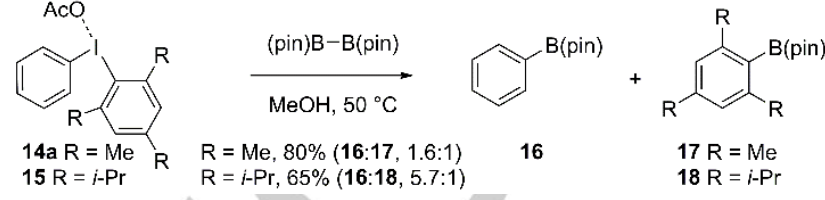

Scheme 4. Aryl transfer selectivity with boron nucleophile.

When enolate-type nucleophiles are used, a prominent electronic effect on aryl transfer selectivity is observed (Scheme 5). ${ }^{[9]}$ Olofsson and co-workers have carried out a thorough investigation of chemoselectivity trends of several nucleophiles, including enolates. ${ }^{[9 a]}$ In this landmark mechanistic study, they noted that inclusion of electron-donating methoxy groups onto one of the aryl groups (19a and 20a; Scheme 5) pushes the nucleophile to the comparatively more electron-deficient phenyl group (21: 22, 13:1 and only 21; Scheme 5). Notably, the trimethoxyphenyl group as an auxiliary (20) yielded exclusively 21 albeit in moderate yield (44\%; Scheme 5). Carbon nucleophiles, like boron nucleophiles, display an "anti-ortho" effect with respect to steric effects (Scheme 5). ${ }^{[9 a]}$ When $\mathbf{1 4 b}$, which has a phenyl and mesityl aryl group, reacts with malonate, nitro alkane, and oxazolidinone-derived enolate with selective transfer of the phenyl group over the mesityl group. Diethyl malonate $\mathbf{2 4}$ as nucleophile was phenylated exclusively with $14 \mathrm{~b}$ to yield 21 in 55\% (Scheme 5). Nitroalkane 25 as nucleophile lead to a 6: 1 ratio of 28: 29 favoring phenyl transfer (Scheme 5). ${ }^{[9 \mathrm{c}]}$ Oxazolidinone $\mathbf{2 6}$ as nucleophile produced a 10: 1 mixture of $\mathbf{3 0}$ : 31 with the major product arising from phenyl transfer (Scheme 5). ${ }^{[9 b]}$ Olofsson has also recently demonstrated that pyridyl groups transfer selectively over mesityl groups to malonate nucleophiles, further supporting an anti-ortho effect. ${ }^{[3]}$ However, a confounding report provided by Manetsch and co-workers ${ }^{[9 d]}$ described preferential transfer of the mesityl group over phenyl from $14 \mathrm{~b}$ to ethyl acetoacetate derived nucleophiles, suggesting the need for continued study in this area.

Gaunt and co-workers have reported a novel reaction which merges the aryl transfer ability of diaryliodonium salts and the umpolung-inducing ability of $\mathrm{N}$-heterocyclic carbene catalysts to turn aldehydes into acyl anions. ${ }^{[10]}$ While, the majority of examples were conducted with symmetrical diaryliodonium salts, they explored unsymmetrical salts to transfer a pyridyl group. Consistent with other carbon nucleophiles, an anti-ortho effect was observed when $\mathbf{3 3}$ bearing a mesityl group was used (Scheme 6). Exclusive pyridyl transfer was observed though, in this case, the yield of $\mathbf{3 4}$ was moderate (30\% of $\mathbf{3 4}$, Scheme 6 ). Notably, a uracil-derived "dummy" ligand proved effective in 


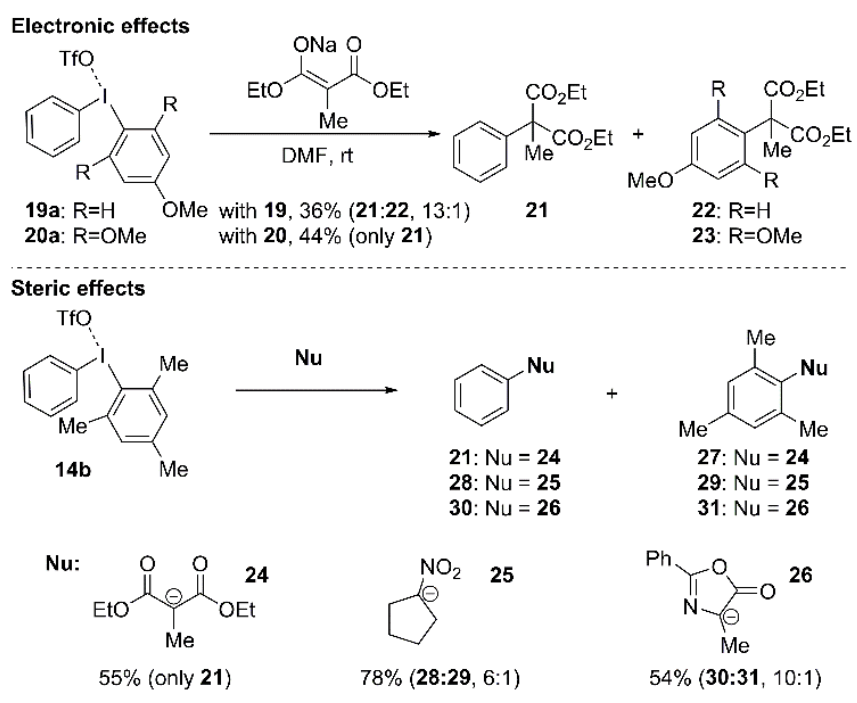

Scheme 5. Aryl transfer selectivity with enolate-type carbon nucleophiles.

increasing the yield and maintaining transfer of the pyridyl group. ${ }^{[10]}$<smiles>O=Cc1nc2ccccc2s1</smiles>

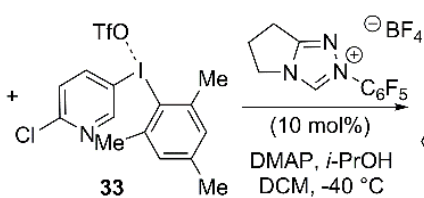
$33 \mathrm{Me} \mathrm{DCM},-40^{\circ} \mathrm{C}$

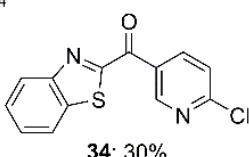

(only aryl transfer product observed)

Scheme 6. Aryl transfer selectivity with acyl anion equivalents.

Ackermann and co-workers have efficiently arylated indole heterocycles with diaryliodonium salts under metal-free conditions (Scheme 7). [11] An electronic effect is observed wherein 19b transfers the less electron-rich phenyl group to indole 35, yielding a 1.5:1 ratio of 36: $37 \mathrm{C} 3$-arylated indole products (Scheme 7). While the electronic effect observed in this case (1.5: 1 ) is more modest than that observed with malonate nucleophiles ( 10: 1), it is consistent with the more electron-deficient aryl group being transferred to the nucleophile. Additionally, Ackermann and co-workers also noted an anti-ortho effect with indole nucleophiles. ${ }^{[11]}$
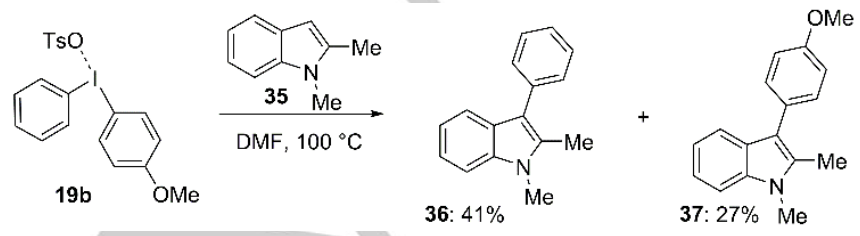

Scheme 7. Aryl transfer selectivity with indole nucleophiles.
In summary, arylation of $C$-nucleophiles with unsymmetrical diaryliodonium salts favors transfer of the comparatively less electron-rich aryl group and the less sterically hindered aryl group (anti-ortho effect). Given these observations both electron-rich and sterically bulky aryl groups could function as broadly applicable "dummy" ligands on the iodane. Several examples of this approach are provided in scheme $8 .{ }^{[12]}$ Dohi and Kita have used a 4-methoxyphenyl (anisyl) group as a "dummy" ligand on 38 in order to transfer the boron-containing aryl group to malonate 39 to yield $55 \%$ of $\mathbf{4 0}$ (Scheme 8 ). ${ }^{12 a]}$ The mesityl (Mes) group also serves as an efficient "dummy" group in unsymmetrical diaryliodonium salt $\mathbf{4 1}$, and thereby preferentially transfers the 4bromophenyl moiety to oxindole $\mathbf{4 2 ^ { [ 1 2 b ] }}$ and cyanoacetate $\mathbf{4 4 ^ { [ 1 2 c ] }}$ nucleophiles to provide 43 and 45 in $65 \%$ and $64 \%$ yield, respectively (Scheme 8). Unsymmetrical aryl(Mes)iodonium salts are efficient reagents for selective aryl transfer to $C$-nucleophiles and are readily accessed by known synthetic methods. ${ }^{[3 b]}$ Future developments with $C$-nucleophiles should be continued with the mesityl "dummy" group and begin to explore other opportunities that exploit electronic effects.

Electronic control with an electron-rich auxiliary
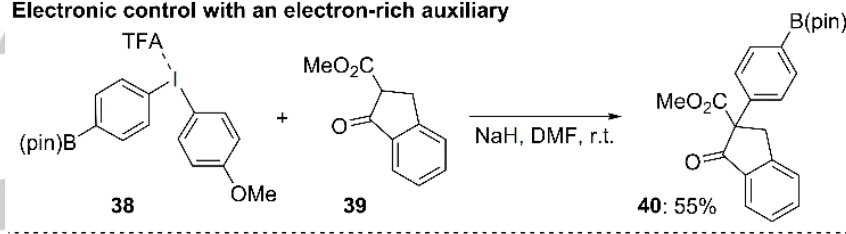

Steric control with sterically bulky auxiliary

$\mathrm{TfO}$<smiles>Cc1cc(C)c(I(O)c2ccc(Br)cc2)c(C)c1</smiles>

41<smiles>Cc1cc(C)c(I(O)c2ccc(Br)cc2)c(C)c1</smiles>

41

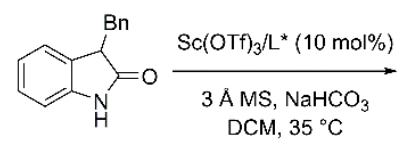

42<smiles>O=C1Nc2ccccc2C1(Br)c1ccc(Br)cc1</smiles>

43: $65 \%, 94 \%$ ee
Scheme 8. Additional examples of selective aryl transfer from unsymmetrical diaryliodonium salts to carbon nucleophiles.

\subsection{Nitrogen nucleophiles}

Aryl amines are common motifs in pharmaceuticals and agrochemicals and diaryliodonium salts offer an attractive approach to their metal-free synthesis. A range of amine nucleophiles have been used in selective coupling reactions with unsymmetrical diaryliodonium salts, including amides, cyanamides, hydroxamic acids, nitrite, and $\mathrm{N}$-heterocycles. Electronic and steric effects of the diaryliodonium aryl groups are the dominant factors that influence selectivity in the cases presented below. 
Amides $^{[13]}$ and cyanamides ${ }^{[14]}$ react with unsymmetrical diaryliodonium salts $19 \mathrm{a}$ and 49 , respectively, with pronounced electronic control (Scheme 9). Specifically, Olofsson and coworkers $^{[13]}$ have demonstrated that the phenyl group of $19 \mathrm{a}$ preferentially transfers to acetanilide $\mathbf{4 6}$ over the anisyl group (Scheme 9). While the yield of $\mathbf{4 7}$ is moderate (45\%), the aryl transfer selectivity is excellent as only trace quantities $(<5 \%)$ of 48 are observed (Scheme 9). Additionally, Zhang and coworkers $^{[14]}$ observed that the reaction of $\mathbf{4 9}$ with cyanamide $\mathbf{5 0}$ resulted in high yield and excellent selectivity for transfer of the electron-deficient 4-nitrophenyl group (51, 92\%) over the phenyl group $(\mathbf{5 2},<5 \%$; Scheme 9$)$. Steric effects also have a prominent influence on aryl transfer selectivity in these reactions and an ortho-effect is observed in these cases. The reaction of $46^{[13]}$ or hydroxamic acid $\mathbf{5 4} \mathbf{4}^{[15]}$ with $\mathbf{1 4 b}$ leads to preferential transfer of the mesityl group in both cases (Scheme 9). However, note that the ratio of aryl transfer depends on the nucleophile. A 4: 1 ratio of 53: 47 was observed with amide 46 and a 2.5: 1 ratio of 55: 56 was observed with $\mathbf{5 4}$ (Scheme 9).

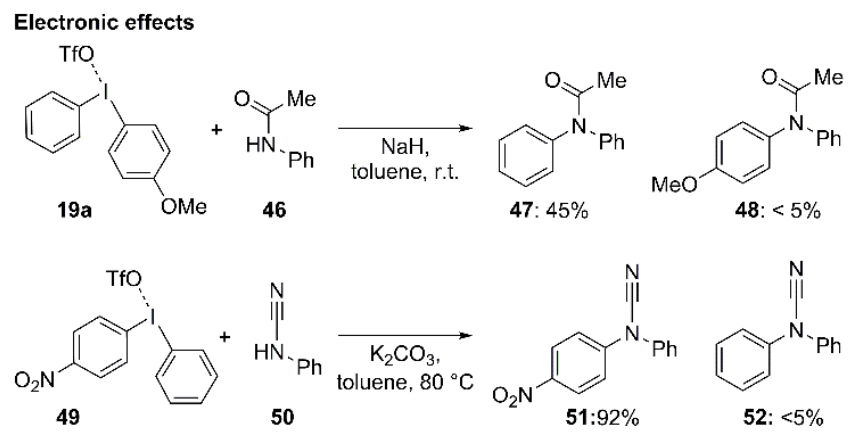

$$
\text { Steric effects }
$$

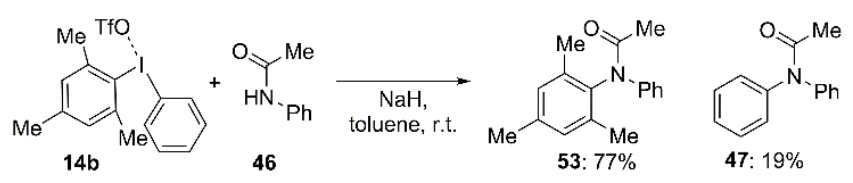

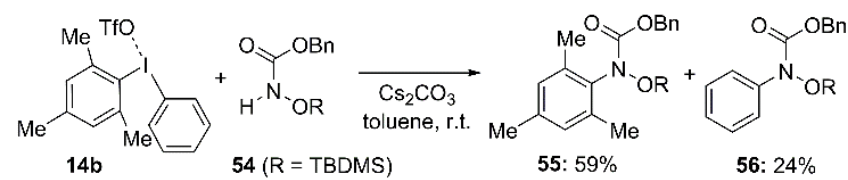

Scheme 9. Aryl transfer selectivity with amide, cyanamide, and hydroxamic acid nucleophiles.

Novak and co-workers have studied the aryl transfer selectivity of unsymmetrical diaryliodonium salts to nitrogencontaining heterocycles by experimental and computational methods. ${ }^{[16]}$ Electronic and steric effects consistent with other $N$ nucleophiles were observed (Scheme 10). Specifically, the reaction of diaryliodonium salt $\mathbf{4 9}$ with pyrazole $\mathbf{5 7}$ resulted in exclusive transfer of the 4-nitrophenyl group and high yield (86\%) of 58 (Scheme 10); this selectivity was also observed with cyanamide (Scheme 9). Notably, under these reaction conditions and with pyrazole $\mathbf{5 7}$ as nucleophile, steric effects have greater influence than electronic effects in aryl transfer selectivity. When unsymmetrical diaryliodonium salt $\mathbf{5 9}$, which has 4-nitrophenyl and mesityl groups, reacts with pyrazole 57 there is a 3: 1 preference for transfer of the mesityl group $(60)$ over the electrondeficient 4-nitrophenyl group (Scheme 10).

\section{Electronic effects}

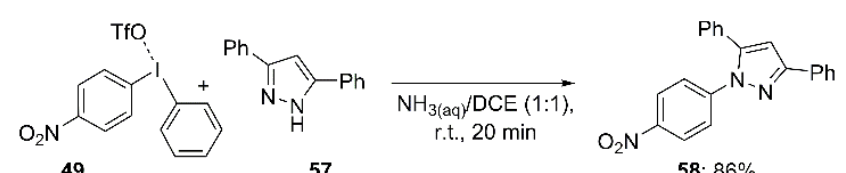

$$
\text { Steric effects }
$$

Scheme 10. Aryl transfer selectivity with pyrazole nucleophiles.

Sodium nitrite has recently been used as a nucleophile with diaryliodonium salts as a method to access synthetically versatile nitroarenes. ${ }^{[17]}$ The electronic and steric effects observed for aryl group transfer from unsymmetrical salts are consistent with those observed for other $\mathrm{N}$-nucleophiles (Scheme 11). Specifically, a ratio of $\sim 14: 1$ is observed for transfer of the more electron deficient phenyl group from 19a and almost exclusive transfer of the more sterically bulky mesityl group is observed from $14 \mathbf{b}$ (Scheme 11).
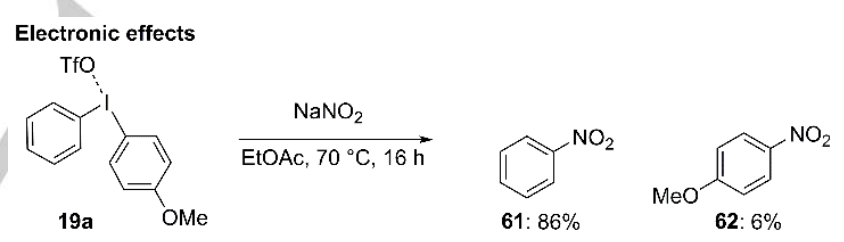

Steric effects

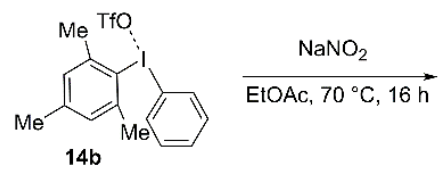<smiles>Cc1cc(C)c([N+](=O)[O-])c([N+](=O)[O-])c1</smiles><smiles>O=[N+]([O-])c1ccccc1</smiles>

Scheme 11. Aryl transfer selectivity with nitrite nucleophile.

Collectively, nitrogen nucleophiles undergo reductive ligand coupling with the more electron-deficient group when steric effects are absent and with the more sterically encumbered group for 2,6-substituted aryl groups. ${ }^{[18]}$ Consistent with these observations, Sandtorv and Stuart have used the trimethoxyphenyl group as an extremely electron-rich aryl "dummy" ligand to transfer electron-deficient aryl groups to alicyclic amine nucleophiles $\left(64 \rightarrow 66\right.$; Scheme 12). ${ }^{[18 a]}$ Additionally, Muñiz and co-workers have effectively used a simple 
Electronic effects

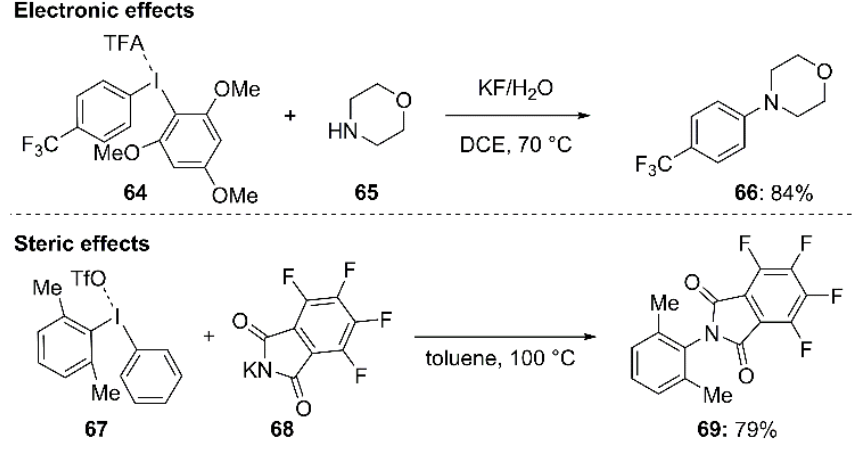

Scheme 12. Additional examples of selective aryl transfer from unsymmetrical diaryliodonium salts to nitrogen nucleophiles.

phenyl group as a "dummy" ligand for the selective transfer of 2,6disubstituted aryl groups to phthalimide nucleophiles $(67 \rightarrow 69$; Scheme 12), which provides access to sterically encumbered aniline derivatives. ${ }^{[18 \mathrm{~b}]}$

\subsection{Oxygen and other chalcogen nucleophiles}

Group 16 nucleophiles, particularly oxygen, have been the most prominent in nucleophile arylation with diaryliodonium salts that proceed through a $\lambda^{3}$-iodane intermediate. A broad range of oxygen nucleophiles are compatible in these reactions, including those derived from phenol, carboxylic acids, carbamates, and hydroxyl imides. Similar to nitrogen nucleophiles, reactions with oxygen-derived nucleophiles are sensitive to electronic and steric effects, in which an ortho-effect is observed in the latter.

Phenol nucleophiles react with diaryliodonium salts to produce industrially important diaryl ethers; synthetic and mechanistic studies have been described for these reactions. ${ }^{[9 a],[19]}$ Olofsson has reported a chemoselectivity study of unsymmetrical diaryliodonium salts that included phenol nucleophiles. ${ }^{[9 a]}$ A large electronic effect was observed for the reaction of 19a with phenol 70 under basic conditions (Scheme 13). Similar to malonate and amide nucleophiles, phenol nucleophiles coupled to the less electron-rich phenyl group with high selectivity and yield of 71 (94\%; Scheme 13). Likewise, 4quinolinone $\mathbf{7 3}$ reacts with $\mathbf{4 9}$ under basic conditions and the electron-deficient 4-nitrophenyl moiety transfers exclusively and in high yield (74, 77\%; Scheme 13). ${ }^{[19 c]}$ The steric effect that is observed in reactions of diaryliodonium salts with phenol nucleophiles favors transfer of the more sterically encumbered aryl group (i.e., an ortho effect; Scheme 13). The ratio for products 75: 71 is $\sim 1.9: 1$ which is less than that observed with nitrogen nucleophiles (compare Scheme 9 and 13).

Carboxylate and related carbamate nucleophiles react with diaryliodonium salts at higher temperatures than are required for phenol nucleophiles. ${ }^{[19 a, b],[20]}$ However, similar electronic and steric effects are noted with these $O$-nucleophiles. For example, diaryliodonium salts $19 \mathrm{a}$ and 49 , which have been used to bench-

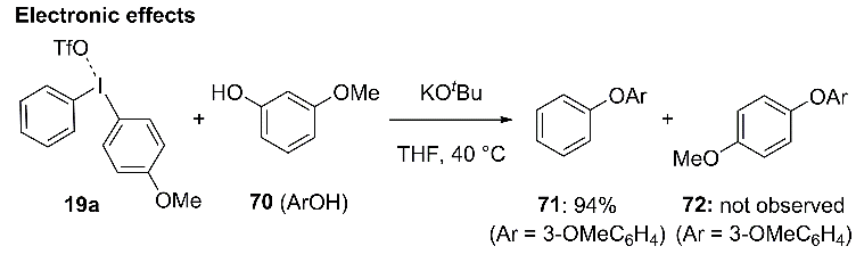

(necN, $60^{\circ} \mathrm{C}$

Steric effects

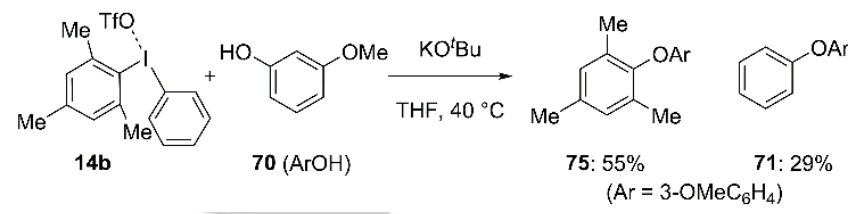

Scheme 13. Aryl transfer selectivity with phenol nucleophiles.

mark electronic effects in other systems (Scheme 9 and 13), both transfer the less electron-rich aryl group to carboxylate and carbamate nucleophiles derived from 76 and 79 (Scheme 14). Additionally, an ortho-effect is observed for reactions of $\mathbf{6 b}$ or $\mathbf{8 2}$ with 76 or $\mathbf{7 9}$, respectively, and product ratios of 2-3: 1 are observed with preference for the aryl group bearing a substituent in the 2-position (Scheme 14). Notably, the very sterically bulky 2,4,6-tri-iso-propylphenyl is transferred with complete selectivity over a phenyl group with carboxylate nucleophiles. ${ }^{[19 a, b]}$
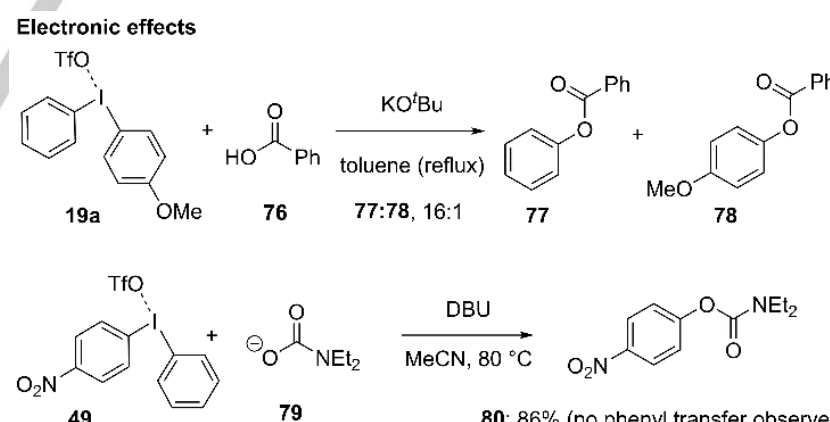

\section{Steric effects}

Me TfO
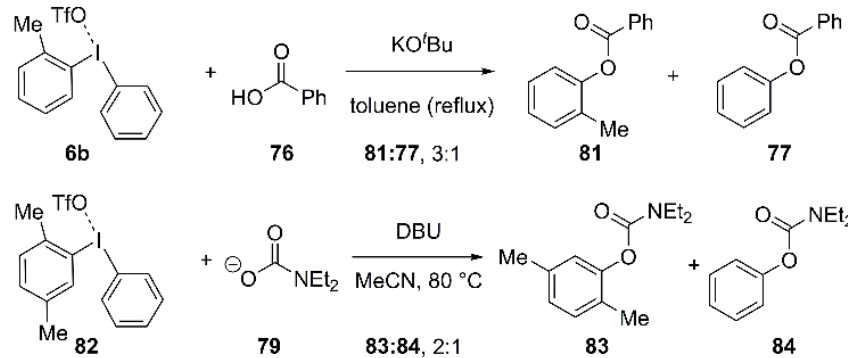

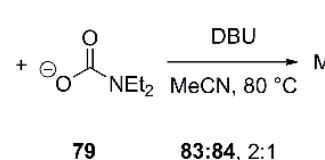

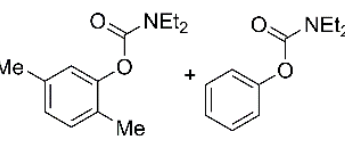

Scheme 14. Aryl transfer selectivity with carboxylate-type nucleophiles. 
Other, less common, oxygen nucleophiles have also been arylated with diaryliodonium salts (i.e., 85 and $\mathbf{8 8}$ (Scheme 15). ${ }^{[11]}$ Electronic effects that are consistent with phenolate and carboxylate nucleophiles are observed. For instance, Olofsson and co-workers have observed that hydroxyimides react with unsymmetrical aryl(TMP)iodonium salt 20a to provide exclusive phenyl group transfer and high yield of 86 (89\%; Scheme 15). ${ }^{[21 a]}$ Similar electronic effects were noted in the arylation of $N$ hydroxybenzo[1,2,3]-triazinone $\mathbf{8 8}$ with unsymmetrical diaryliodnium salt 87 (Scheme 15).[21b] Notably, exclusive selectivity was observed for transfer of the 4-methoxyphenyl group and the thiophene heterocycles was not transferred (Scheme 15). A modest ortho-effect was observed in the reaction of $\mathbf{6 b}$ with $\mathbf{8 5}$ (Scheme 15). Specifically, a 1.2: 1 ratio of $\mathbf{9 0 :} \mathbf{8 6}$ was observed with slight preference for transfer of the 2methylphenyl group (Scheme 15).[21a] Comparatively, when benzoate was used as the nucleophile a 3: 1 preference for transfer of the 2-methylphenyl group was observed (see Scheme 14).

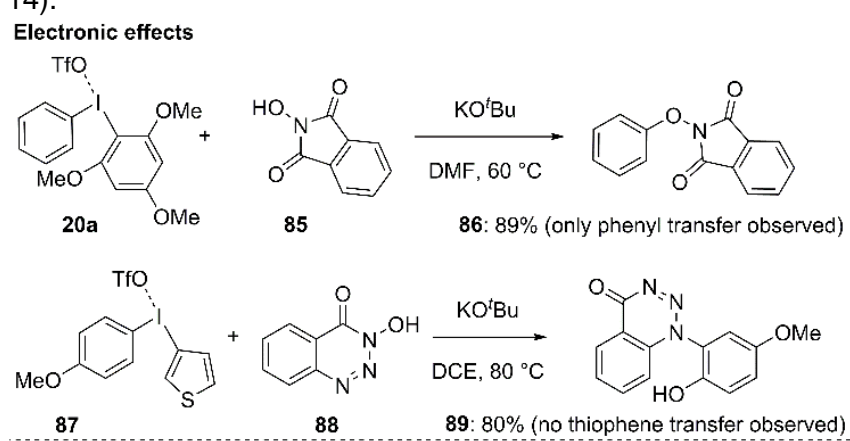
Steric effects<smiles>COc1ccccc1[IH]OCCO</smiles>

6b

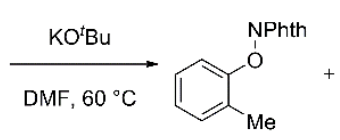

90: $47 \%$

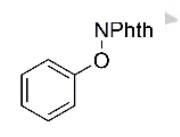

86: $38 \%$
Scheme 15. Aryl transfer selectivity with $N$-hydroxyphthalimide and hydroxamic acid nucleophiles.

Further developments with oxygen nucleophiles may take advantage of both electronic and steric effects in the selection of "dummy" ligands. Several examples are presented below that demonstrate the synergism or competition of these effects in the $O$-arylation of aliphatic alcohols, which is currently an active area of research (Scheme 16). ${ }^{[22]}$ Specifically, Olofsson and coworkers have achieved the arylation of 1-pentanol 91 under basic conditions and observed that the 4-nitrophenyl group is transferred in high yield from $\mathbf{4 9}$ to produce alkyl aryl ether $\mathbf{9 2}$ in $89 \%$ yield (Scheme 16$){ }^{[22 a]}$ Sterically congested alkyl aryl ethers have been synthesized in high yield (95, 86\%; Scheme 16) by Olofsson and co-workers by employing a TMP "dummy" ligand and transferring a 2,6-disubstituted aryl moiety from 93 to sodium tert-butoxide 94 (Scheme 16). ${ }^{[22 c]}$ Sundalam and Stuart demonstrated that when unsymmetrical diaryliodonium salt $\mathbf{5 9}$ reacts with sec-butoxide 96 as nucleophile, the 4-nitrophenyl

\section{Electronic effects}

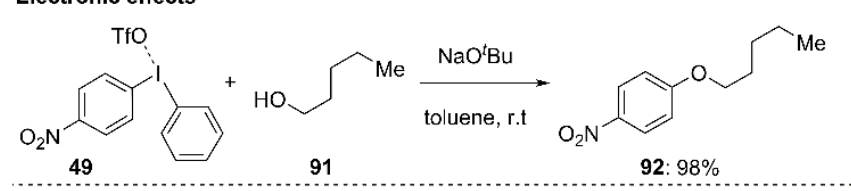

Synergistic electronic and steric effects

Me OTf

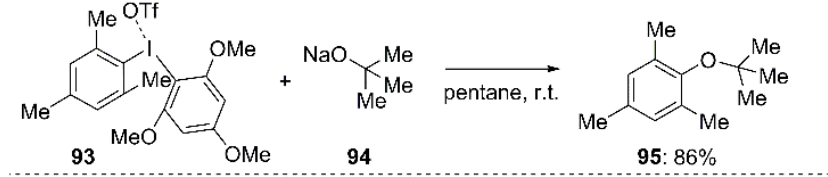

Competitive electronic and steric effects

作

Scheme 16. Additional examples of selective aryl transfer from unsymmetrical diaryliodonium salts to oxygen nucleophiles.

group is transferred preferentially to provide 97 in high yield ( $80 \%$, Scheme 16). ${ }^{[22 b]}$ The aryl transferred selectivity inferred from the reaction of $\mathbf{5 9}$ with alkoxide $\mathbf{9 6}$ is opposite that observed in the reaction of $\mathbf{5 9}$ with $N$-nucleophile, pyrrazole (Scheme 10).

The lower chalcogens, such as sulfur, selenium, and tellurium have been arylated with diaryliodonium salts. Notably, when sulfur nucleophiles are used, the aryl transfer selectivity varies dramatically with the nature of the sulfur nucleophile. ${ }^{[23]}$ Specifically, the reaction of thiophenol derivatives 99 with unsymmetrical diaryliodonium salt $\mathbf{9 8}$ under basic conditions displays a significant electronic effect wherein the less electronrich 4-chlorophenyl group is transferred (100: 101 is 11: 1, Scheme 17). ${ }^{[23 c]}$ However, when diphenyl thioether 102 reacts with 49 under forcing thermal conditions, the sulfonium product 104 that incorporates the more electron-rich phenyl group is the major product (Scheme 17). ${ }^{[23 b]}$ This is in stark contrast to all other cases presented here in which $\mathbf{4 9}$ selectively transfers the electron-deficient 4-nitrophenyl group (Scheme 9, 10, 13, 14, and 16). A significant ortho-effect is also observed for the reaction of diaryliodonium salt 105 with sodium phenylsulfite (Scheme 17).

\subsection{Halide nucleophiles}

The arylation of halogen nucleophiles with diaryliodonium salts has been dominated by fluorine and in particular fluorine-18 $\left({ }^{18} \mathrm{~F}\right)$ for positron emission tomography (PET) imaging. ${ }^{[24]}$ Selected examples of fluorine arylation are presented that demonstrate aspects of aryl transfer selectivity in the context of both electronic and steric effects. lodide has also appeared as a nucleophile and briefly discussed here as well. 


$$
\text { Electronic effects }
$$

Scheme 17. Selectivity of aryl group transfer from unsymmetrical diaryliodonium salts to sulfur derived nucleophiles.

Electronic and steric effects for aryl transfer selectivity from unsymmetrical diaryliodonium salts that are observed with fluoride nucleophiles are consistent with those of other heteroatom nucleophiles. Specifically, Pike and co-workers have observed a modest electronic and steric effect for diaryliodonium salts 108 and $\mathbf{6 b}$, respectively (Scheme 18). ${ }^{[25 a]}$ In these cases, the more electron-deficient and more sterically encumbered aryl group were transferred preferentially, yielding 109 and 111 as the major products, respectively (Scheme 18). Notably, salt 108 may benefit from both electronic and steric effects in preferential transfer of the 2-bromophenyl group. By extension, an extremely large tri-iso-propylsilyl group in the para-position has even led to modest sterically controlled selectivity with a bromide nucleophile. ${ }^{[26]}$ Given the need to transfer large, biologically active molecules to radio-labelled fluorine for PET imaging, novel "dummy" ligands have been designed for aryl transfer under metal-free conditions. ${ }^{[25 b, c]}$ DiMagno and co-workers ${ }^{[25 b]}$ have designed and demonstrated the utility of cyclophane-containing 112 in fluorine arylation and Liang and co-workers ${ }^{[25 c]}$ have independently designed spiro-iodonium ylides $\mathbf{1 1 4}$ for the same purpose (Scheme 18).

The direct electrophilic iodination of arenes $\left(S_{E} A r\right)$ with NIS or $\mathrm{I}_{2}$ leads to mixtures of para- and ortho-substitution products in ratios that range from 1.2:1 to 6:1. ${ }^{[27]}$ DiMagno and co-workers have developed a high-yielding route to iodo arenes that involves the intermediacy of an unsymmetrical diaryliodonium salt 116. ${ }^{[27]}$ While aryl transfer selectivity is not an issue in this case, this work is included here because it employs an unsymmetrical diaryliodonium salt as a general approach to a synthetic challenge and delivers higher para-selectivity (para: ortho, 9: 1) than is observed in electrophilic iodination mentioned previously (Scheme 19).

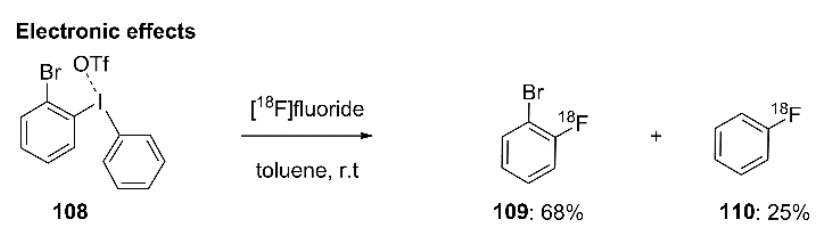

Steric effects

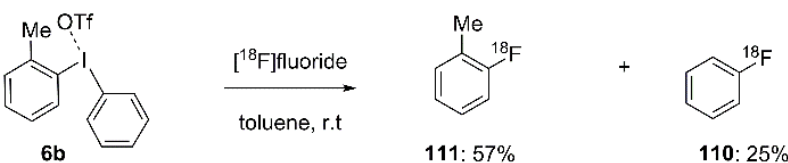

Other auxiliaries<smiles>COc1ccc(I(F)c2cccc(C34CC5CC(CC(C5)C3)C4)c2OC)cc1</smiles>

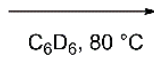<smiles>COc1ccc(F)cc1</smiles>

113: $81 \%$<smiles>CCC1(C)OC(=O)C(=Ic2ccc(OC)cc2)C(=O)O1</smiles>

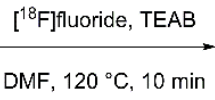<smiles>COc1ccc(F)cc1</smiles>

113: $15 \%(\mathrm{RCY})$

Scheme 18. Selectivity of aryl group transfer to fluoride and bromide nucleophiles nucleophiles.

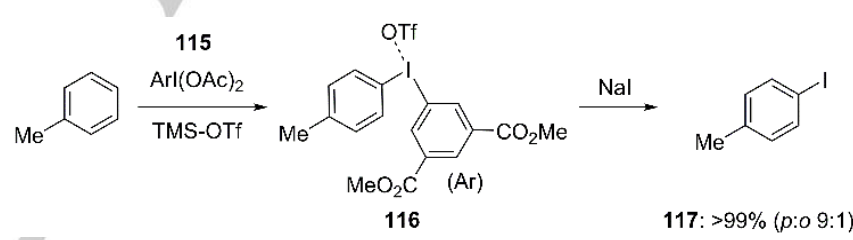

Scheme 19. Arene iodination enabled by an unsymmetrical diaryliodonium salt.

\subsection{General trends in aryl transfer selectivity}

Exploiting $\lambda^{3}$-iodane intermediates for nucleophile arylation under metal-free conditions is a novel approach that accommodates a diverse range of nucleophiles. As noted above, electronic and steric effects are the dominant control factors that lead to selective aryl group transfer from unsymmetrical diaryliodonium salts in these reactions. Collectively, some interesting periodic trends are observed for nucleophiles derived from the first row of the $p$-block. First, in general, the more electron-deficient aryl group is transferred to the attacking nucleophile, though an exception to this was noted for diaryl thioether nucleophiles. Product ratios based on this effect appeared more modest at either end of the $p$ block (i.e., $B$ - and $F$-nucleophiles) than in the center (i.e., $C$-, $N$-, and $O$-nucleophiles; Scheme 20). Steric effects are more nucleophile dependent than electronic effects, yet still displayed a periodic trend. An anti-ortho effect was observed for $B$ - and $C$ nucleophiles, and this was more prominent for $C$-nucleophiles (Scheme 20). The transition from $C$ - to $N$-nucleophiles, for the 
most part, marked a change from anti-ortho to ortho effect in which transfer of the more sterically encumbered aryl group was preferred (blue in Scheme 20). Additionally, this effect was most pronounced for $\mathrm{N}$-nucleophiles and decreased toward $\mathrm{F}$ nucleophiles (Scheme 20). Taking the properties of the attacking nucleophile into account, aryl transfer selectivity based on steric effects generally follows Hard-Soft Acid-Base (HSAB) theory. Soft nucleophiles display an anti-ortho effect and hard nucleophiles display an ortho effect; this is also inclusive of aryl transfer selectivity to metals, such as $\mathrm{Cu}(\mathrm{I})$ and $\mathrm{Pd}(\mathrm{II}){ }^{[1]}$ On the basis of these observations, general "dummy" ligands that incorporate strong electronic effects would likely tolerate the broadest range of aryl groups and nucleophiles. However, the transfer of sterically congested aryl groups may be achieved with relatively simple phenyl "dummy" ligands when hard nucleophiles are used and efforts toward this have already been described. ${ }^{[18 b, 22 c]}$
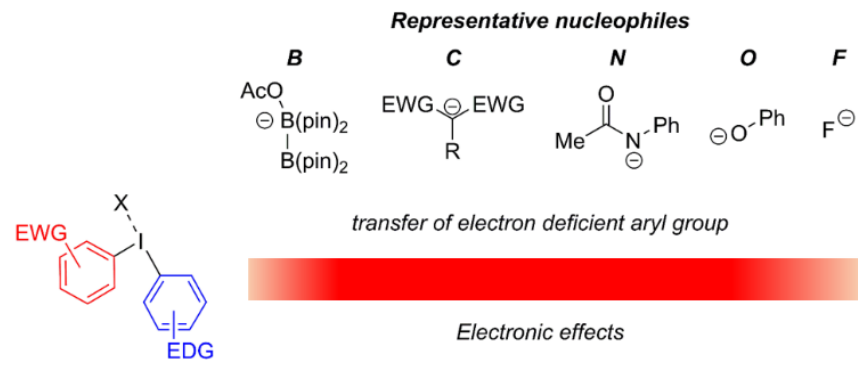

transfer of electron deficient aryl group
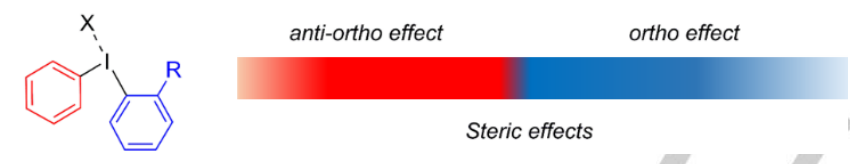

Scheme 20. General trends in aryl transfer selectivity from diaryl- $\lambda^{3}$-iodanes.

\section{Arylation involving $\lambda^{3}$-iodane radical anion intermediates}

The observation of $\lambda^{3}$-iodane radical anion intermediates in reactions of diaryliodonium salts recently emerged in 2010. [28] While relatively few examples involving these intermediates have been described compared to the more common closed-shell $\lambda^{3}$ iodane intermediates, this alternate mechanistic pathway makes previously challenging transformations possible. ${ }^{[29]}$ In these reactions, the diaryliodonium salt reacts with an electron-rich arene via single electron transfer (SET) to form a radical anionradical cation pair $(\mathbf{1 1 9}, \mathrm{Scheme} 21)$. The radical anion-radical cation pair 119 collapses with transfer of the more electron-rich aryl group from the diaryliodonium salt to the incoming simple arene (118, Scheme 21). The selectivity of aryl transfer from 20b under this mechanistic paradigm is opposite to that observed from the closed-shell reactivity of $\lambda^{3}$-iodanes (see Scheme 5 ). In this case a simple phenyl group or other more electron-deficient aryl group may serve as a "dummy" ligand and this has been used to effect selective transfer of electron-rich heterocycles to incoming simple arenes (121-123; Scheme 21).

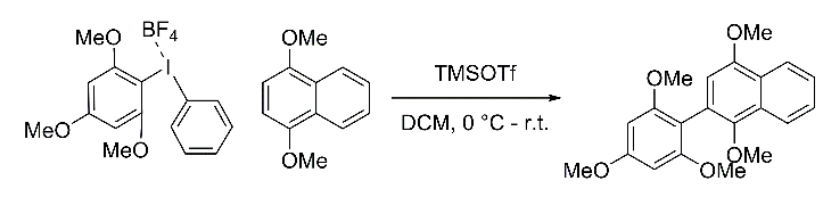

$20 \mathrm{~b}$

118

120: $74 \%$

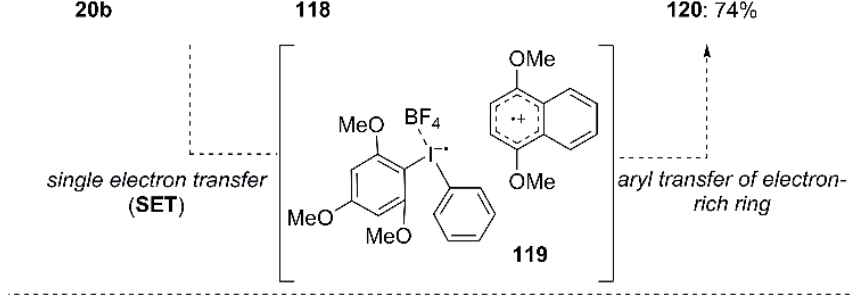

$\overbrace{\mathrm{OMe}}^{\mathrm{TMSOTf}}$<smiles>COc1cc(Br)c(-c2cc(C)cs2)c(OC)c1</smiles><smiles>COc1ccc(-c2cc(C)c(C)o2)c(OC)c1</smiles>

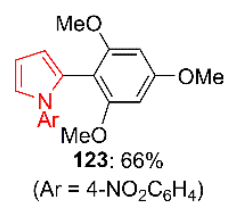

Scheme 21. Selective aryl transfer from unsymmetrical diaryl- $\lambda^{3}$-iodane radical anions.

\section{Arylation involving aryl radical intermediates}

Aryl radical intermediates participate in a broad range of transformations and the burgeoning field of photo-redox catalysis provides a steady stream of inspiring transformations. Diaryliodonium salts extrude aryl radicals under a variety of conditions and are mediated by reagents or catalysts (Scheme 22). In general, the aryl transfer selectivity by extrusion of an aryl radical is more modest and less clearly defined than aryl transfer selectivity in ionic reactions; this is true for both electronic and steric effects. ${ }^{[30]}$

$\stackrel{\text { reagent or catalyst }}{\longrightarrow}$ R

Scheme 22. General reaction involving an aryl radical derived from a diaryliodonium salt.

Several different aryl radical traps have been used in synthetic settings that form C-C and C-heteroatom bonds. In three of the four examples presented in Scheme 23, the less electron-rich phenyl group is preferentially extruded as a radical from unsymmetrical diaryliodonium salt 19a. The exception to this is the arylation of benzoquinone wherein a slight bias for transfer of the more electron-rich 4-methoxyphenyl group is observed. [30b] 
In all cases the selectivity is modest and ranges from $~ 1.5: 1$ to $\sim$ : 1 in the best case (Scheme 23).
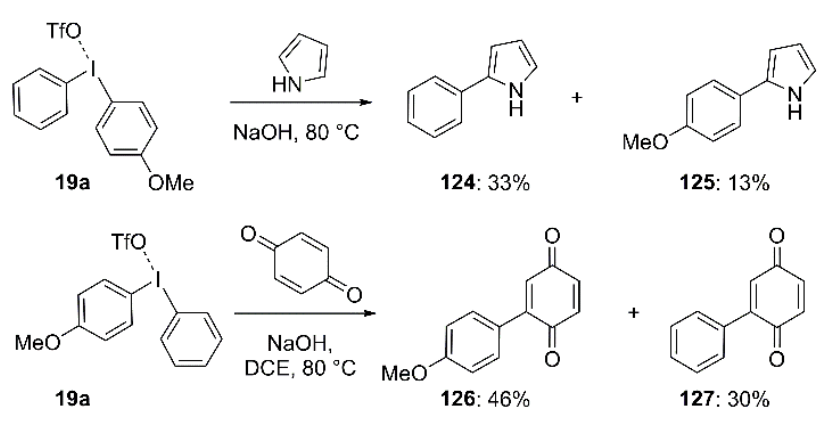

TfO
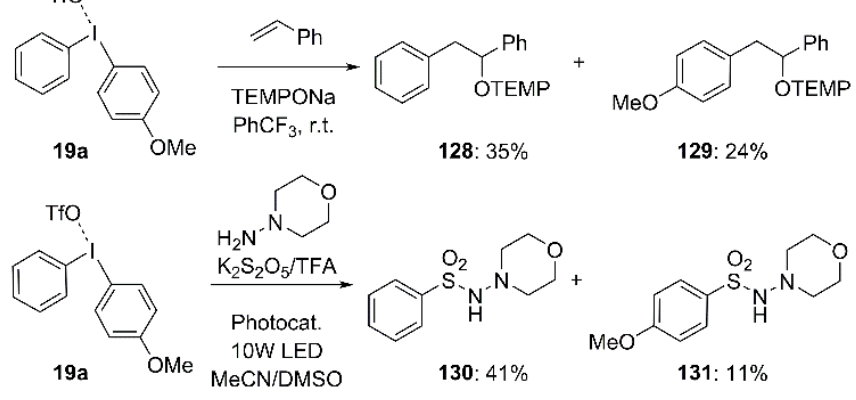

Scheme 23. Electronic effects in aryl transfer selectivity by extrusion of aryl radicals.

The aryl transfer selectivity observed for extrusion of aryl radicals also varies with respect to steric effects (Scheme 24). The arylation of benzoquinone with $6 \mathrm{~b}$ via the proposed intermediacy of an aryl radical displayed a modest ortho effect (132: 127 is 2: 1, Scheme 24). ${ }^{[30 \mathrm{~b}]}$ However, the extrusion of an aryl radical from $14 \mathrm{~b}$ and trapping with either styrene ${ }^{[30 c]}$ or a hydrazine ${ }^{[30 d]}$ derivative displayed a modest anti-ortho effect (Scheme 24). The difference in reaction conditions may indeed be a source of disparate aryl radical extrusion in these cases. However, continued mechanistic characterization of emerging reactions is also likely to provide more insight into the controlling factors that dictate the selectivity of aryl radical extrusion from unsymmetrical diaryliodonium salts.

\section{Arylation involving aryne intermediates}

Aryne intermediates derived from diaryliodonium salts have been known since the early 1970's, ${ }^{[31]}$ and early examples were initiated by ortho-C-H deprotonation. However, in 1999 Kitamura and co-workers replaced the triflate leaving group in the Kobayashi reagent with a phenyliodonium leaving group. ${ }^{[32]}$ While, this is an exceedingly mild approach to benzynes, translation to substituted arynes remains limited due to the synthetic sequence required to generate (phenyl)[o-(trimethylsilyl)aryl]iodonium triflate. A C-H deprotonation approach to arynes derived from unsymmetrical aryl(auxiliary)iodonium salts has the potential to

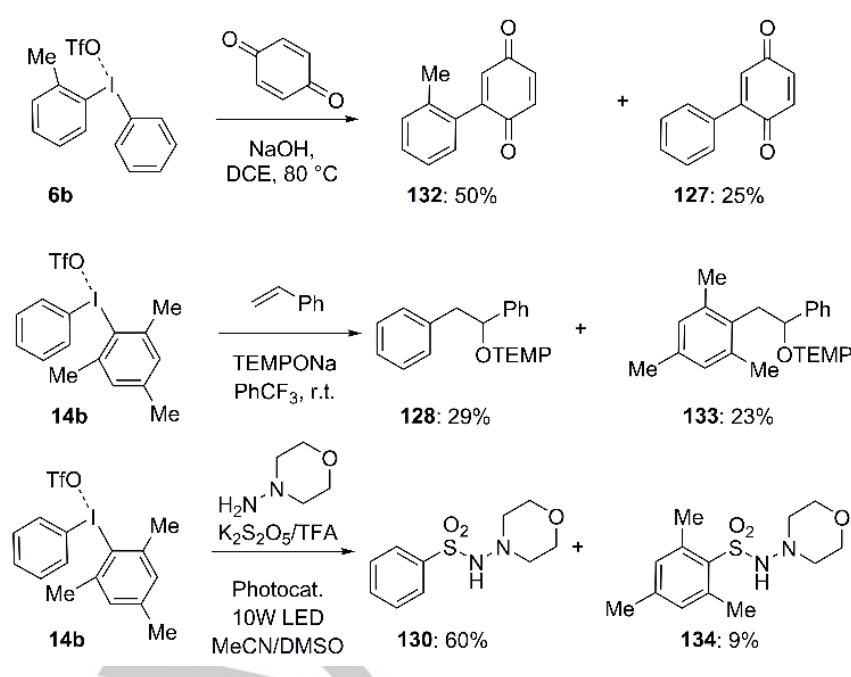

Scheme 24. Steric effects in aryl transfer selectivity by extrusion of aryl radicals.

dramatically expand substrate scope to highly functionalized arynes and recent examples highlight this emerging approach. ${ }^{[33]}$ This is primarily a result of the relative ease that unsymmetrical diaryliodonium salts may be synthesized from simple arenes, aryl iodides, and arylboronic acid building blocks. ${ }^{[2]}$

In both of the recent examples a mesityl group is an effective "dummy" ligand because the 2- and 6-positions are blocked which forces deprotonation and aryne extrusion at the other aryl group (Scheme 25). Sundalam et al. recently described this approach and applied it to extrusion of highly functionalized arynes that were trapped with furan in a cycloaddition reaction $(\mathbf{1 3 5} \rightarrow \mathbf{1 3 6}$; Scheme 25). ${ }^{[33 a, c]}$ Wang and Huang independently described an arylation of amide nucleophiles that proceeds through an aryne intermediate. In this case a mixture of regioisomers is observed as a result of the poor directing effects of a 4-bromo group on an aryne $(135 \rightarrow 137+138$; Scheme 25$) .{ }^{[33 b]}$ The synthetic utility of arynes and the high yield and selectivity of extrusion from aryl(Mes)iodonium salts is likely to engender further studies with these reagents.

\section{Conclusions and outlook}

Unsymmetrical diaryliodonium salts participate in a wide range of metal-free transformations that involve $\lambda^{3}$-iodanes, $\lambda^{3}$-iodane radical anions, aryl radicals, and arynes. Despite the diversity of these reactive intermediates, general trends for aryl transfer selectivity have been organized on the basis of electronic and steric effects of the iodonium reagent's aryl groups. While the electronic and steric effects are dependent on both reactive intermediate and the nature of the functional group undergoing arylation, several trends are observed. For the most part, 


\section{References}

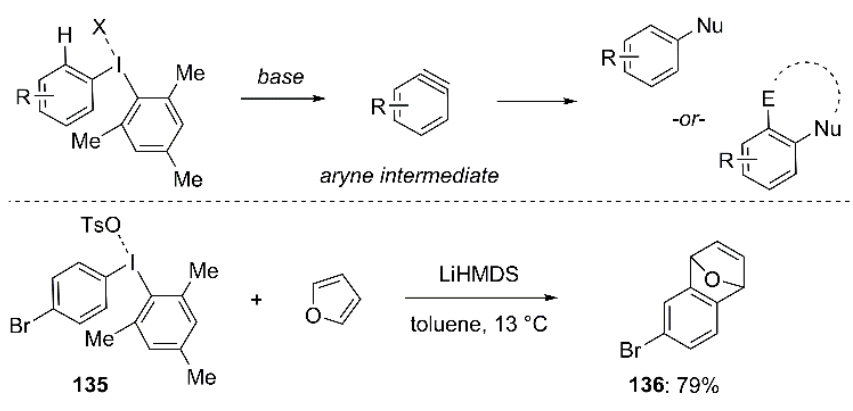<smiles>Cc1cc(C)cc(I(O[Tl])c2ccc(Br)cc2)c1</smiles>

135

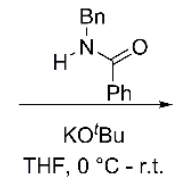

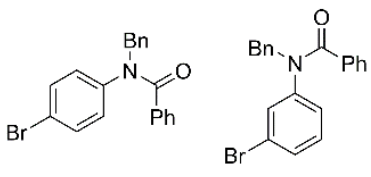

138: $22 \%$
Scheme 25. Selective aryl transfer by extrusion of arynes.

transfer of the more electron-deficient aryl group is favored, except for reactions involving $\lambda^{3}$-iodane radical anions. Additionally, for $\lambda^{3}$-iodanes an anti-ortho effect is observed for nucleophiles based on early p-block elements ( $B$ and $C$ ), but an ortho-effect is observed for the later p-block elements $(\mathrm{N}, \mathrm{O}, \mathrm{F})$. Ortho-blocked aryl groups obviate $\mathrm{C}-\mathrm{H}$ deprotonation and therefore function as useful "dummies" for the extrusion of arynes from unsymmetrical diaryliodonium salts because they push ortho $\mathrm{C}-\mathrm{H}$ deprotonation to the other aryl group. General conclusions regarding the steric effects involving other reactive intermediates, such as aryl radicals, are premature at this stage though this is an exciting area of chemistry to watch evolve.

Considering these trends, the development of novel "dummy" ligands that are based on strong electronic effects would appear to be the most general strategy forward. Moreover, we posit that the broader synthesis community will benefit significantly from reaction discovery and development studies that use general unsymmetrical aryl(auxiliary)iodonium reagents. Such studies will build upon previous work and further advance aryl transfer selectivity that is paramount to broad adoption of these reagents in synthesis.

\section{Acknowledgements}

Portland State University is acknowldeged for financial support. The reviewers are acknowledged for detailed and insightful comments on the initial submission of this work.

Keywords: hypervalent iodine $\cdot$ diaryliodonium $\cdot$ arylation $•$ chemoselectivity $\cdot$ metal-free
[1] (a) N. R. Deprez, M. S. Sanford Inorg. Chem. 2007, 46, 1924. (b) E. A Merritt, B. Olofsson Angew. Chem. Int. 2009, 48, 9052. (c) B. Olofsson Top. Curr. Chem. 2016, 373, 135. (d) K. Aradi, R. L. Tóth, G. Tolnai, Z. Novák Synlett 2016, 27, 1456.

[2] Based on an April 24, 2017 SciFinder search for "diaryliodonium".

[3] For selected examples, see: (a) A. McKillop, D. Kemp Tetrahedron 1988, 45, 3299. (b) M. Ochiai, M. Toyonari, T. Sueda, Y. Kitagawa Tetrahedron 1996, 37, 8421. (c) A. Shah, V. W. Pike, D. A. Widdowson J. Chem. Soc., Perkin Trans. 1997, 2463. (d) M. D. Hossain, Y. Ikegami, T. Kitamura J. Org. Chem. 2006, 71, 9903 - 9905. (e) M. D. Hossain, T. Kitamura Bull. Chem. Soc. Jpn. 2007, 80, 2213 - 2219. (f) M. Bielawski, B. Olofsson Chem. Commun. 2007, 2521. (g) M. Bielawski, M. Zhu, B. Olofsson Adv. Syn. Catal. 2007, 349, 2610. (h) M. Zhu, N. Jalalian, B. Olofsson Synlett, 2008, 592. (i) M. Bielawski, D. Aili, B. Olofsson J. Org. Chem. 2008, 73 4602. (j) M. Bielawski, B. Olofsson Org. Synth. 2009, 86, 308. (k) E. A. Merritt, J. Malmgren, F. J. Klinke, B. Olofsson Synlett 2009, 2277. (I) M. Bielawski, J. Malmgren, L. M. Pardo, Y. Wikmark, B. Olofsson ChemistryOpen 2014, 3, 19. (m) L. Qin, B. Hu, K. D. Neumann, E. J. Linstad, K. McCauley, J. Veness, J. J. Kempinger, S. G. DiMagno Eur. J. Org. Chem. 2015, 5919. (n) T. L. Seidl, S. K. Sundalam, B. McCullough, D. R. Stuart J. Org. Chem. 2016, 81, 1998. (0) V. Carreras, A. H. Sandtorv, D. R. Stuart J. Org. Chem. 2017, 82, 1279.

[4] (a) P. J. Stang, V. V. Zhdankin Chem. Rev. 1996, 96, 1123. (b) V. V. Zhdankin, P. J. Stang Chem. Rev. 2002, 102, 2523. (c) T. Wirth Hypervalent lodine Chemistry: Modern Developments in Organic Synthesis, Springer: Berlin, 2003. (d) V. V. Zhdankin, P. J. Stang Chem. Rev. 2008, 108, 5299. (e) V. V. Zhdankin Hypervalent lodine Chemistry: Preparation, Structure, and Synthetic Applications Wiley: Chichester, 2013. (f) A. Yoshimura, V. V. Zhdankin Chem. Rev. 2016, 116, 3328 (g) Hypervalent lodine Chemistry, T. Wirth, Ed. Top. Curr. Chem. 2016.

[5] H. Pinto de Magalhães, H. P. Lüthi, A. Togni Org. Lett. 2012, 14, 3830.

[6] F. M. Beringer, A. Brierley, M. Drexler, E. M. Gindler, C. C. Lumpkin J. Am. Chem. Soc. 1953, 75, 2708.

[7] (a) Y. Yamada, M. Okawara Bull. Chem. Soc. Jpn. 1972, 45, 1860. (b) K. M. Lancer, G. H. Wiegand J. Org. Chem. 1976, 41, 3360.

[8] N. Miralles, R. M. Romero, E. Fernández, K. Muñiz Chem. Commun. 2015, 51, 14068.

[9] (a) J. Malmgren, S. Santoro, N. Jalalian, F. Himo, B. Olofsson Chem. Eur J. 2013, 19, 10334. (b) Z. Chai, B. Wang, J.-N. Chen, G. Yang Adv. Synth. Catal., 2014, 356, 2714. (c) C. Dey, E. Lindstedt, B. Olofsson Org Lett. 2015, 17, 4554 . (d) A. Monastyrskyi, N. K. Namelikonda, R Mantesch J. Org. Chem. 2015, 80, 2513-2520.

[10] Q. Y. Toh, A. McNally, S. Vera, N. Erdmann, M. J. Gaunt J. Am. Chem Soc. 2013, 135, 3772.

[11] L. Ackermann, M. Dell'Acqua, S. Fenner, R. Vicente, R. Sandmann Org. Lett. 2011, 13, 2358

[12] For other representative examples of selective aryl transfer to carbon nucleophiles, see: (a) M. Ito, I. Itani, Y. Toyoda, K. Morimoto, T. Dohi, Y. Kita Angew. Chem. Int. Ed. 2012, 51, 12555. (b) J. Guo, S. Dong, Y. Zhang, Y. Kuang, X. Liu, L. Lin, X. Feng Angew. Chem. Int. Ed. 2013, 52, 10245. (c) X. Qian, J. Han, L. Wang Adv. Synth. Catal. 2016, 358, 940.

[13] F. Tinnis, E. Stridfeldt, H. Lundberg, H. Adolfsson, B. Olofsson Org. Lett. 2015, 17, 2688.

[14] J. Li, X. Zheng, W. Li, W. Zhou, W. Zhu, Y. Zhang New. J. Chem. 2016 40, 77.

[15] Y. Yang, X. Wu, J. Han, S. Mao, X. Qian, L. Wang Eur. J. Org. Chem. 2014, 6854 .

[16] (a) Z. Gonda, Z. Novák Chem. Eur. J. 2015, 21, 16801. (b) T. Bihari, B. Babinszki, Z. Gonda, S. Kovács, Z. Novák, A. Stirling J. Org. Chem. 2016, $81,5417$.

[17] M. Reitti, P. Villo, B. Olofsson Angew. Chem. Int. Ed. 2016, 55, 8928 8932. 
[18] (a) A. H. Sandtorv, D. R. Stuart Angew. Chem. Int. Ed. 2016, 55, 15812 (b) N. Lucchetti, M. Scalone, S. Fantasia, K. Muñiz Angew. Chem. Int Ed. 2016, 55, 13335

[19] (a) T. B. Petersen, R. Khan, B. Olofsson Org. Lett. 2011, 13, 3462 - 3465. (b) N. Jalalian, T. B. Petersen, B. Olofsson Chem. Eur. J. 2012, 18 14140. (c) E. Lindstedt, R. Ghosh, B. Olofsson Org. Lett. 2013, 15, 6070 (d) P. D. Nahide, C. R. Solorio-Alvarado Tetrahedron Lett. 2017, 58, 279.

[20] W. Xiong, C. Qi, Y. Peng, T. Guo, M. Zhang, H. Jiang Chem. Eur. J. 2015 $21,14314$.

[21] (a) R. Ghosh, B. Olofsson Org. Lett. 2014, 16, 1830. (b) W.-M. Shi, X.P. Ma, C.-X. Pan, G.-F. Su, D.-L. Mo J. Org. Chem. 2015, 80, 11175. (c) R. Ghosh, E. Stridfeldt, B. Olofsson Chem. Eur. J. 2014, 20, 8888- 8892

[22] (a) R. Ghosh, E. Lindstedt, N. Jalalian, B. Olofsson ChemistryOpen 2014, 3, 54. (b) S. K. Sundalam, D. R. Stuart J. Org. Chem. 2015, 80, 6456. (c) E. Lindstedt, E. Stridfeldt, B. Olofsson Org. Lett. 2016, 18, 4234.

[23] (a) N. Umierski, G. Manolikakes Org. Lett. 2013, 15, 188. (b) L. Racicot, T. Kasahara, M. A. Ciufolini Org. Lett. 2014, 16, 6382. (c) D. Wang, X. Yu, K. Zhao, L. Li, Y. Ding Tetrahedron Lett. 2014, 55, 5739.

[24] M. S. Yusubov, D. Y. Svitich, M. S. Larkina, V. V. Zhdankin ARKIVOC, 2013 (i), $364-395$.

[25] (a) J.-H. Chun, S. Lu, Y.-S. Lee, V. W. Pike J. Org. Chem. 2010, 75, 3332. (b) J. W. Graskemper, B. Wang, L. Qin, K. D. Neumann, S. G. DiMagno Org. Lett. 2011, 13, 3158. (c) B. H. Rotstein, N. A. Stephenson, N. Vasdev, S. H. Liang Nat. Commun. 2014, 5, 4365.

[26] M. S. Yusubov, D. Y. Svitich, A. Yoshimura, B. J. Kastern, V. N. Nemykin, V. V. Zhdankin Eur. J. Org. Chem. 2015, 4831 - 4834.
[27] B. Hu, W. H. Miller, K. D. Neumann, E. J. Linstad, S. G. DiMagno Chem. Eur. J. 2015, 21, 6394.

[28] T. Dohi, M. Ito, N. Yamaoka, K. Morimoto, H. Fujioka, Y. Kita Angew. Chem. Int. Ed. 2010, 49, 3334.

[29] (a) N. Yamoaka, K. Sumida, I. Itani, H. Kubo, Y. Ohnishi, S. Sekiguchi, T. Dohi, Y. Kita Chem. Eur. J. 2013, 19, 15004. (b) K. Morimoto, Y Ohnishi, D. Koseki, A. Nakamura, T. Dohi, Y. Kita Org. Biomol. Chem. 2016, 14, 8947. (c) K. Morimoto, A. Nakamura, T. Dohi, Y. Kita Eur. J. Org. Chem. 2016, 4294

[30] (a) J. Wen, R.-Y. Zhang, S.-Y. Chen, J. Zhang, X.-Q. Yu J. Org. Chem 2012, 77, 766. (b) D. Wang, B. Ge, L. Li, J. Shan, Y. Ding J. Org. Chem. 2014, 79, 8607. (c) M. Hartmann, Y. Li, C. Mück-Lichtenfeld, A. Stude Chem. Eur. J. 2016, 22, 3485. (d) N.-W. Liu, S. Liang, G. Manolikakes Adv. Synth. Catal. 2017, 359, 1308.

[31] (a) T. Akiyama, Y. Imasaki, M. Kawanisi Chem. Lett. 1974, 229. (b) J. I. G. Cadogan, A. G. Rowley, J. T. Sharp, B. Sledzinski, N. H. Wilson J. Chem. Soc. Perkin Trans. 1 1975, 1072.

[32] T. Kitamura, M. Yamane, K. Inoue, M. Todaka, N. Fukatsu, Z. Meng, Y. Fujiwara J. Am. Chem. Soc. 1999, 121, 11674.

[33] (a) S. K. Sundalam, A. Nilova, T. L. Seidl, D. R. Stuart Angew. Chem. Int. Ed. 2016, 55, 8431. (b) M. Wang, Z. Huang Org. Biomol. Chem., 2016, 14, 10185. (c) D. R. Stuart Synlett 2017, 28, 275. 
WILEY-VCH

\section{MINIREVIEW}

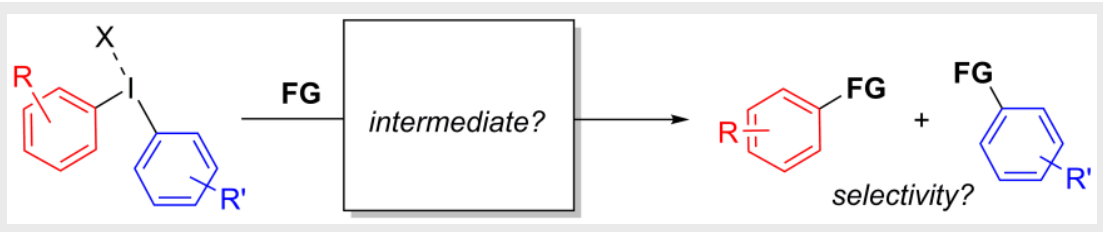

Diaryliodonium salts are novel arylation reagents that participate in diverse chemical transformations. Aryl transfer selectivity from unsymmetrical diaryliodonium salts is examined and organized by the type of reactive intermediate that is formed in the reaction. Electronic and steric effects of the aryl groups are the dominant controlling factors for selective aryl transfer.
David R. Stuart*

Page No. - Page No.

Aryl Transfer Selectivity in Metal-Free Reactions of Unsymmetrical Diaryliodonium Salts 